\title{
Mathematical modeling of radiant heating of a closed rectangular area under conditions of convective heat transfer at the external boundaries
}

\author{
Alexander Nee \\ National Research Tomsk Polytechnic University, Tomsk, Russia
}

\begin{abstract}
Mathematical modeling of radiant heating of a closed rectangular area under conditions of convective heat transfer at the external boundaries is passed. The fields of temperature and stream function, illustrating the unsteady nature of the heat transfer were obtained. The extent influence of convective heat transfer at the external boundaries on the circulating flows formation in the gas cavity are shown.
\end{abstract}

\section{Introduction}

It is known [1] that the use of gas infrared emitters (GIE) contributes to the localization of thermal energy consumption, which allows the efficient use of energy resources to create a comfortable environment in the limited size of volume parts where it is needed (working areas in a large-scale factories). It is shown [2,3] that the study of heat transfer process in a typical heat supply object with radiant heating system is advantageously carried out by mathematical modeling. It should be noted that the models $[2,4]$, based on the heat balance doesn't allow air convection and heat conduction in the enclosure structures. Perspectively to use the approach $[5,6]$, based on a system of unsteady Navier - Stokes and energy equations for gas and heat conduction equation for the solid walls.

An approach [3] for modeling of thermal regimes of the large objects with radiant heating systems which is based on the numerical solution of the vorticity transport, Poisson and energy equations for the air and the heat conduction equation for endlocsure structures are suggested. Problem [3] was solved within the limits of a plane turbulent heat transfer model taking into account the heat dissipation of energy into the wall, but it was assumed that the radiant flux coming from the GIE, evenly distributed only on the lower horizontal boundary interface "air - enclosure structure". Numerical analysis [7] (laminar model) is carried out for the four possible variants of the radiant energy distribution along the internal boundaries of the domain under the assumption that the heat transfer on the external boundaries with the environment is absent. However, part of the thermal energy accumulated by enclosure structures is extracted to the environment. The investigation of heat transfer is preferably carried out with the convective heat exchange at the external boundaries.

The aim of this study is mathematical modeling of a closed rectangular area radiant heating under conditions of convective heat exchange at the external boundaries.

\section{Problem formulation}

Boundary problem of unsteady conjugate heat transfer with the accounting of convective heat exchange at the external boundaries is solved. Solution domain is shown in Figure 1.

Gas cavity of rectangular cross-section bounded by solid conducting walls of finite thickness is considered. At the interfaces "air - enclosure structures" are given equality of temperatures and heat fluxes. It was assumed that the gas is heat-conducting viscous incompressible Newtonian fluid and absolutely transparent for heat radiation, thermal properties of air and enclosure structures materials do not depend on temperature. Flow regime is turbulent. The radiant energy distribution is given by Lambert's law [8]. 


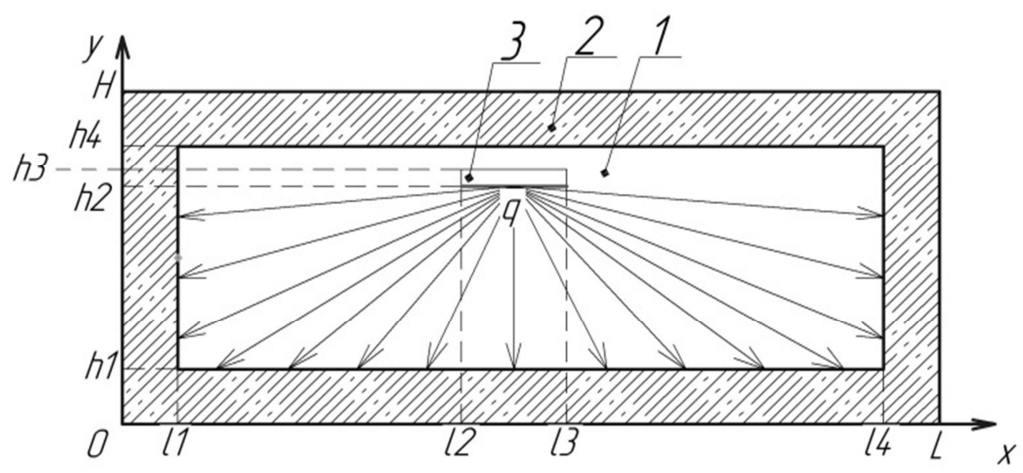

Figure 1. Solution domain: 1 - air; 2 - enclosure structures; 3 - gas infrared radiator (symbolic notation).

The process of turbulent conjugate heat transfer under study is described by unsteady Reynolds and energy equations for gas and the heat conduction equation for enclosure structures. In dimensionless variables "vorticity $\Omega$ - stream function $\Psi$ - temperature $\Theta$ " these equations are as follows [9]:

$$
\begin{gathered}
\frac{\partial \Omega}{\partial \tau}+U \frac{\partial \Omega}{\partial X}+V \frac{\partial \Omega}{\partial Y}=\frac{\partial^{2}}{\partial X^{2}}\left[\left(\sqrt{\frac{\operatorname{Pr}}{R a}}+v_{t}\right) \Omega\right]+\frac{\partial^{2}}{\partial Y^{2}}\left[\left(\sqrt{\frac{\operatorname{Pr}}{R a}}+v_{t}\right) \Omega\right]+\frac{\partial \Theta_{1}}{\partial X}, \\
\nabla^{2} \Psi=-\Omega, \\
\frac{\partial \Theta_{1}}{\partial \tau}+U \frac{\partial \Theta_{1}}{\partial X}+V \frac{\partial \Theta_{1}}{\partial Y}=\frac{\partial^{2}}{\partial X^{2}}\left[\left(\frac{1}{\sqrt{\operatorname{Ra} \cdot \operatorname{Pr}}}+\frac{v_{t}}{\operatorname{Pr}_{t}}\right) \Theta_{1}\right]+\frac{\partial^{2}}{\partial Y^{2}}\left[\left(\frac{1}{\sqrt{\operatorname{Ra} \cdot \operatorname{Pr}}}+\frac{v_{t}}{\operatorname{Pr}_{t}}\right) \Theta_{1}\right], \\
\frac{\partial \Theta_{2}}{\partial F o_{2}}=\nabla^{2} \Theta_{2},
\end{gathered}
$$

Eddy viscosity was determined by using a model of Baldwin - Lomax [10]:

$$
v_{t}=l_{m}^{2}|\Omega|, l_{m}=k y,
$$

where $l_{m}$ - mixing length; y - coordinate; $k$ - Karman constant.

Initial conditions for equations (1) - (4) are as follows:

$$
\begin{gathered}
\Psi(X, Y, 0)=0 ; \Omega(X, Y, 0)=0 ; U(X, Y, 0)=0 ; V(X, Y, 0)=0 ; \\
\Theta_{1}(X, Y, 0)=\Theta_{2}(X, Y, 0)=0 .
\end{gathered}
$$

The boundary conditions at the external boundaries of the solution domain are as follows:

$$
\begin{gathered}
X=0,0<Y \leq 1, Y=1,0<X \leq 1,2: \frac{\partial \Theta_{2}(X, Y, \tau)}{\partial n}=B i \cdot\left(\Theta_{e}-\Theta\right) . \\
X=1,2,0<Y \leq 1, Y=0,0<X \leq 1,2: \frac{\partial \Theta_{2}(X, Y, \tau)}{\partial n}=0 ;
\end{gathered}
$$


at the internal interfaces "solid wall - air", parallel to the axis OX:

$$
\Psi=0, \frac{\partial \Psi}{\partial Y}=0,\left\{\begin{array}{l}
\Theta_{i}=\Theta_{j}, \\
\frac{\partial \Theta_{i}}{\partial Y}=\frac{\lambda_{j}}{\lambda_{i}} \cdot \frac{\partial \Theta_{i}}{\partial Y}+K i,
\end{array} \quad \text { где } \mid \begin{array}{l}
i=\overline{1,2} \\
j=\overline{1,2}
\end{array}\right.
$$

at the internal interfaces "solid wall - air", parallel to the axis OY:

$$
\Psi=0, \frac{\partial \Psi}{\partial X}=0,\left\{\begin{array}{l}
\Theta_{i}=\Theta_{j}, \\
\frac{\partial \Theta_{i}}{\partial X}=\frac{\lambda_{j}}{\lambda_{i}} \cdot \frac{\partial \Theta_{i}}{\partial X}+K i,
\end{array} \quad \text { где } \mid \begin{array}{l}
i=\overline{1,2} \\
j=\overline{1,2}
\end{array}\right.
$$

where где $F o$ - Fourier number; $R a-$ Rayleigh number; $K i-$ Kirpichev number; $\operatorname{Pr}-\operatorname{Prandtl}$ number; $\mathrm{Pr}_{t}-$ the eddy Prandtl number; Bi - Biot number; X,Y - dimensionless coordinates corresponding $\mathrm{x}, \mathrm{y} ; \tau$ - dimensionless time; $\mathrm{U}, \mathrm{V}$ - dimensionless velocity corresponding to $\mathrm{u}, \mathrm{v}$;

Equations (1) - (4) with the corresponding initial and boundary conditions are solved by finite difference method, as in [11]. The using solution method was tested on a model problem [12]. Comparison of the results (temperature fields) with [12] showed their good agreement.

\section{The results of numerical modeling}

Numerical study conducted at values of dimensionless criteria corresponding to turbulent flow: $\mathrm{Ra}=10^{9}, \operatorname{Pr}=0,71, \operatorname{Pr}_{t}=1,0<\mathrm{Ki} \leq 30$. The fields of temperature and stream functions for different values of dimensionless time are shown in Figure 2. Isolines current with the sign "-" oriented clockwise, with the sign "+" against.
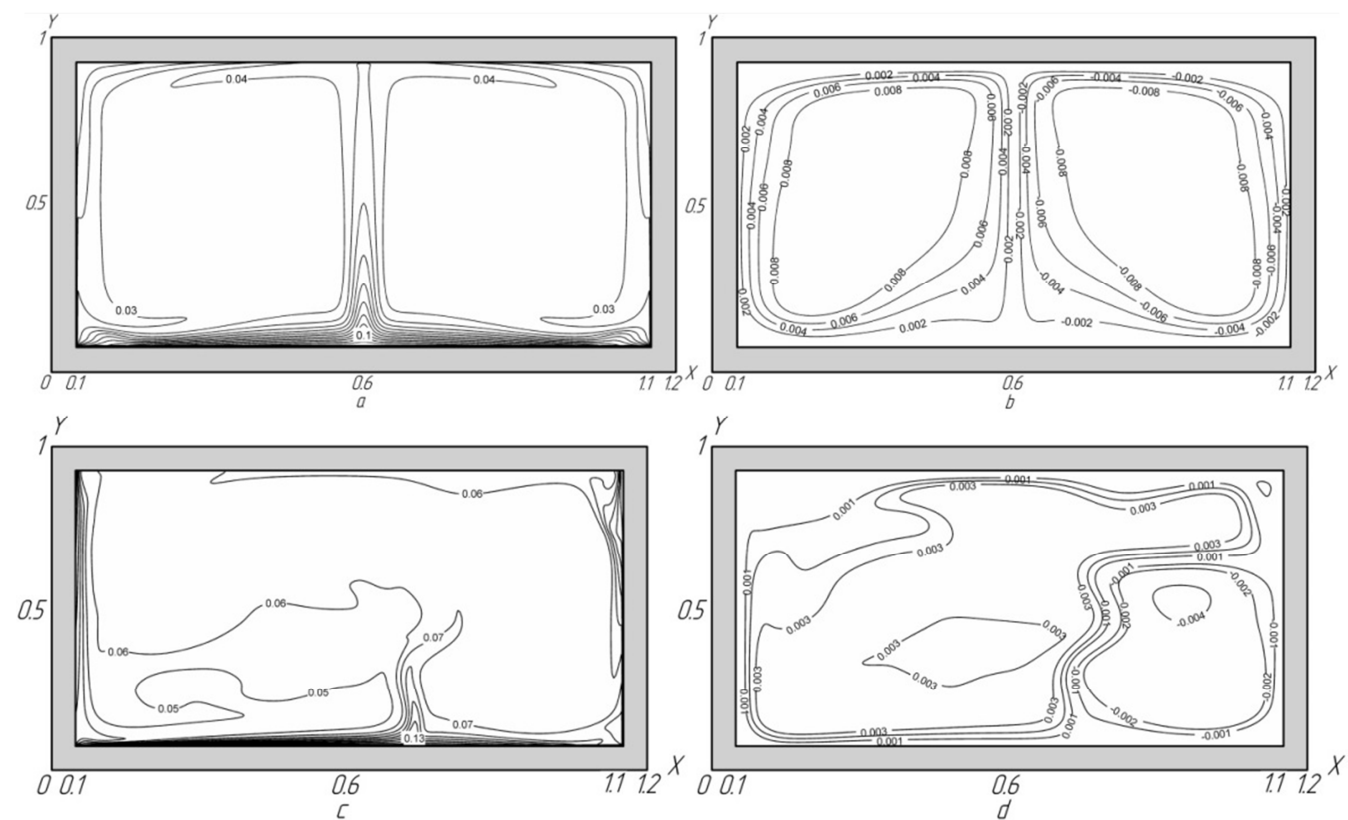

Puc. 2. Isolines of temperatures $(a, b)$ and stream functions $(c, d)$ at $\tau=1000$ :

$a, b)$ Heat insulation at the external boundaries; $c, d)$ Heat exchange with environment at the external boundaries.

Figure 2 shows, that heat exchange at the external boundaries leads to deformation of the convective torch and the modification of vortex flows in the gas cavity. At $\tau=1000$ in conditions of heat insulation at the external boundaries (fig. $2 \mathrm{a}$, b) all energy coming from GIE is accumulated by 
air and enclosure structures. Symmetrical temperature and stream functions fields are formed. The upward flow of the heated air is displaced towards the right wall in the case of the heat sink on the external boundaries (Fig. 2 c, d). One large-scale convective cell and small recirculating flow in the lower right corner are formed in the gas cavity.
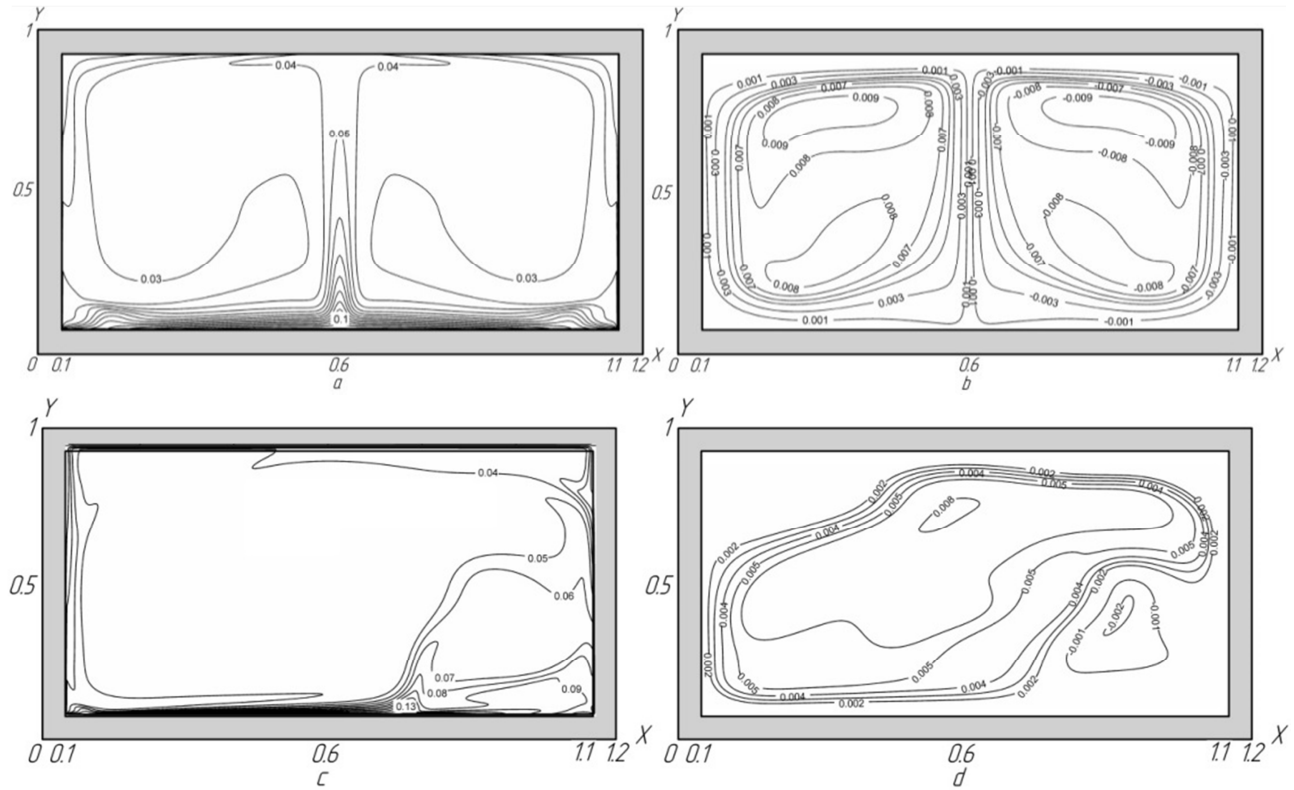

Puc. 3. Isolines of temperatures $(a, b)$ and stream functions $(c, d)$ at $\tau=1500$ :

$a, b)$ Heat insulation at the external boundaries; $c, d)$ Heat exchange with environment at the external boundaries.

The eddy currents are modified with increasing time to $\tau=1500$ (Fig. 3 b, d). The scale of the secondary vortex is reduced (Figure $3 \mathrm{~g}$ ), which is associated with a decrease in temperature of the left wall. Thermal torch in section $\mathrm{X}=0.7$ significantly shifted in the direction of the $\mathrm{X}$-axis, which is caused by the influence of convective heat exchange at the external boundaries of the solution domain. The isotherm shape corresponding to the dimensionless temperature $\Theta=0,06$ repeats the boundary between the vortex structures. At the same time essentially inhomogeneous temperature fields are formed, which is associated with intense heat sink at the external boundaries and the size of the solution domain corresponding to turbulent flow regime.

\section{Conclusion}

According to the results of a numerical study it is found that in conditions of the radiant heating of a closed rectangular area filled with gas and limited by solid conducting walls are formed essentially unsteady fields of temperatures and stream functions. The convective heat transfer at the external boundaries leads to the modification of the circulating currents of air. It can be concluded that at problem formulation of conjugate heat transfer with radiant sources of energy is advisable to take into account the energy loss to the environment.

Scientific supervisor: Head of Thermal Theory and Engineering Department of the Energy Institute of the National Research Tomsk Polytechnic University, Doctor of Physical and Mathematical Sciences Kuznetsov G.V.

This study is the part of the Research Works of the State publisher "Nauka” №13.1339.2014/K (code of the target-oriented scientific-technological program 2.1410.2014) 


\section{References}

1. K. Roth, ASHRAE, 6 (2007)

2. V. V. Buhmirov, S. A. Krupennikov, Yu. S. Solnyshkova, Her. of Ivan. St. Pow. Un. 4 (2010)

3. G.V. Kuznetsov, V.I. Maksimov, T.A. Nagornova, N.I. Kurilenko, G. Ya. Mamontov, J. of Engin. Ph. and Thermoph. 86, 3 (2013)

4. V. V. Buhmirov, S. A. Krupennikov, Yu. S. Solnyshkova, Her. of Ivan. St. Pow. Un. 2 (2009)

5. G.V. Kuznetsov, M.A. Sheremet, Fl. Dyn. 41 (2006)

6. G.V Kuznetsov, M.A. Sheremet, T. and A. 16, 1 (2009)

7. G.V. Kuznetsov, T.A. Nagornova, A.E. Ni, J. of Engin. Ph. and Thermoph. 88, 1 (2015)

8. V.P. Isachenko Heat transfer (Energy, 1975)

9. H. Sajjadi, R. Kefayatib, Therm. Sc. 19, 1 (2015)

10. T. Cebeci Analysis of turbulent flows with computer programs (Elsevier Ltd, 2013)

11. G.V. Kuznetsov, P.A. Strizhak J. of Engin. Thermoph. 19, 2 (2010)

12. H.N. Dixit, V. Babu Int. J. H. M. Tr. 49 (2006) 